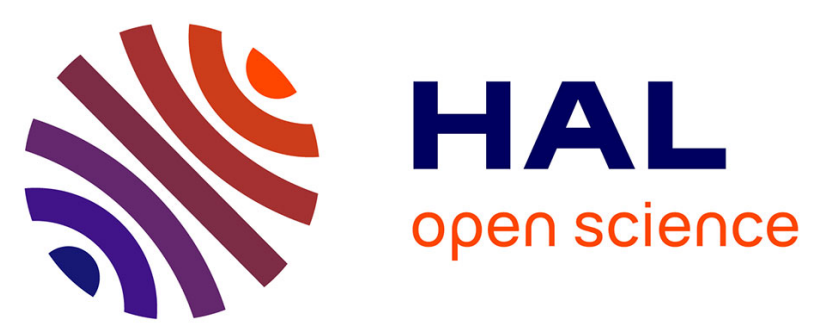

\title{
Optimized Laser Thermal Annealing on Germanium for High Dopant Activation and Low Leakage Current
}

\author{
Maryam Shayesteh, Dan O\&apos; Connell, Farzan Gity, Philip \\ Murphy-Armando, Ran Yu, Karim Huet, Inès Toque-Tresonne, Fuccio \\ Cristiano, Simona Boninelli, Henrik Hartmann Henrichsen, et al.
}

\section{To cite this version:}

Maryam Shayesteh, Dan O\&apos; Connell, Farzan Gity, Philip Murphy-Armando, Ran Yu, et al.. Optimized Laser Thermal Annealing on Germanium for High Dopant Activation and Low Leakage Current. IEEE Transactions on Electron Devices, 2014, 61 (12), pp.4047 - 4055. 10.1109/ted.2014.2364957 . hal-01921394

\section{HAL Id: hal-01921394 \\ https://hal.science/hal-01921394}

Submitted on 13 Nov 2018

HAL is a multi-disciplinary open access archive for the deposit and dissemination of scientific research documents, whether they are published or not. The documents may come from teaching and research institutions in France or abroad, or from public or private research centers.
L'archive ouverte pluridisciplinaire HAL, est destinée au dépôt et à la diffusion de documents scientifiques de niveau recherche, publiés ou non, émanant des établissements d'enseignement et de recherche français ou étrangers, des laboratoires publics ou privés. 


\title{
Optimized Laser Thermal Annealing on Germanium for High Dopant Activation and Low Leakage Current
}

\author{
Maryam Shayesteh, Dan O' Connell, Farzan Gity, Student Member, IEEE, Philip Murphy-Armando, Ran Yu, \\ Karim Huet, Ines Toqué-Tresonne, Fuccio Cristiano, Simona Boninelli, Henrik Hartmann Henrichsen, \\ Peter Folmer Nielsen, Dirch Hjorth Petersen, and Ray Duffy
}

\begin{abstract}
In this paper, state-of-the-art laser thermal annealing is used to fabricate Ge diodes. We compared the effect of laser thermal annealing (LTA) and rapid thermal annealing (RTA) on dopant activation and electrical properties of phosphorus and Arsenic-doped $n+/ p$ junctions. Using LTA, high carrier concentration above $10^{20} \mathrm{~cm}^{-3}$ was achieved in n-type doped regions, which enables low access resistance in $\mathrm{Ge}$ devices. Furthermore, the LTA process was optimized to achieve a diode $I_{\mathrm{ON}} / I_{\mathrm{OFF}}$ ratio $\sim 10^{5}$ and ideality factor $(n) \sim 1.2$, as it allows excellent junction depth control when combined with optimized implant conditions. On the other hand, RTA revealed very high $I_{\mathrm{ON}} / I_{\mathrm{OFF}}$ ratio $\sim 10^{7}$ and $\mathrm{n} \sim 1$, at the cost of high dopant diffusion and lower carrier concentrations which would degrade scalability and access resistance.
\end{abstract}

Index Terms-Ge, laser thermal annealing (LTA), leakage current, $\mathbf{n}+/ \mathbf{p}$ junction.

\section{INTRODUCTION}

$\mathbf{I}$ NDEED, many obstacles and challenges need to be addressed before Ge can become a forefront element in advanced CMOS technology. Realization of n-type ultrashallow junctions with highly activated dopants to maintain low

Manuscript received June 30, 2014; revised October 3, 2014; accepted October 21, 2014. Date of publication November 4, 2014; date of current version December 9, 2014. This work was supported in part by the Science Foundation Ireland under Grant 09/SIRG/I1623 and Grant 09/SIRG/I1621, and in part by the Higher Education Authority Programme for Research in Third-Level Institutions. The review of this paper was arranged by Editor H. Shang.

M. Shayesteh, D. O' Connell, F. Gity, P. Murphy-Armando, and R. Duffy are with the Tyndall National Institute, University College Cork, Cork, Ireland (e-mail: maryam.shayesteh@tyndall.ie; dan.oconnell@tyndall.ie; farzan.gity@tyndall.ie; philip.murphy@tyndall.ie; ray.duffy@tyndall.ie).

R. Yu is with Synopsys (Singapore) Private Limited, 300 Beach Rd, Singapore 199555 (e-mail: rranyu@gmail.com).

K. Huet and I. Toqué-Tresonne are with the Laser Systems and Solutions of Europe (LASSE), Dainippon Screen Group, Gennevilliers 92230, France (e-mail: karim.huet@screen-lasse.com; ines.toque-tresonne@ screen-lasse.com).

F. Cristiano is with the Laboratory for Analysis and Architecture of Systems, Centre National de la Recherche Scientifique, Univeristy of Toulouse, Toulouse 31062, France (e-mail: cfuccio@laas.fr).

S. Boninelli is with MATIS IMM CNR, 95123 Catania, Italy (e-mail: simona.boninelli@ct.infn.it).

H. H. Henrichsen and P. F. Nielsen are with CAPRES A/S, Kongens Lyngby 2800, Denmark (e-mail: hhh@capres.com; pfn@capres.com).

D. H. Petersen is with the Department of Micro- and Nanotechnology, DTU Nanotech, Technical University of Denmark, Kongens Lyngby 2800, Denmark (e-mail: Dirch.Petersen@nanotech.dtu.dk).

Color versions of one or more of the figures in this paper are available online at http://ieeexplore.iee.org.

Digital Object Identifier 10.1109/TED.2014.2364957 access resistance is a well-known roadblock on this journey. Meanwhile, the relatively small bandgap $(0.67 \mathrm{eV})$ of $\mathrm{Ge}$ raises difficulties to minimize the leakage current in pn junctions [1]. Various approaches have been developed to solve these issues among which ultrafast laser thermal annealing (LTA) has drawn a lot of interest [2]-[6]. Providing very limited thermal budgets, LTA has been proven to surpass conventional rapid thermal annealing (RTA) as it boosts activation levels of the dopants well above the equilibrium limit while suppressing dopant diffusion [6]-[9]. Leakage current behavior in $\mathrm{p}+/ \mathrm{n}$ and $\mathrm{n}+/ \mathrm{p}$ Ge junctions has been extensively analyzed [10]-[13]. However, the efficiency of the LTA technique on the control of leakage current in Ge junctions is not fully investigated [14].

The aim of this paper is to systematically compare RTA and LTA with respect to dopant activation and electrical performance of the $\mathrm{n}+/ \mathrm{p}$ junction. Moreover, we aim to explore the tradeoff between dopant activation and leakage current. To fully evaluate these thermal processes, we combine a wide range of material characterization with extensive electrical characterization. In this way, we explore surface roughness and morphology, crystalline integrity, carrier profiling, sheet resistance, and carrier mobility in doped layers, as well as leakage current levels and generation mechanisms.

\section{EXPERIMENTAL DETAILS}

Fig. 1 shows a summary of the process flow in this experiment. Ge (100) wafers (p-type, 0.059-0.088 $\Omega \mathrm{cm}$ ) received standard cleaning prior to either $\mathrm{P}$ or As implant with the dose of $10^{15} \mathrm{~cm}^{-2}$ and the energy of 15 and $28 \mathrm{keV}$, respectively. The $\mathrm{P}$ implantation amorphized the Ge substrate to a depth of $30-35 \mathrm{~nm}$ which is $35-40 \mathrm{~nm}$ for As. It is a feature of Ge substrates that the amorphous/crystalline $(\mathrm{a} / \mathrm{c})$ interface is not smooth after ion implantation. Following implantation, LTA (single pulse, $\lambda=308 \mathrm{~nm}$ ) was applied on one set of samples at $E_{\mathrm{TH}}=0.55 \mathrm{~J} / \mathrm{cm}^{2}, E_{\mathrm{TH}}+0.25$, $E_{\mathrm{TH}}+0.5$, and $E_{\mathrm{TH}}+0.65 \mathrm{~J} / \mathrm{cm}^{2}$ energy densities to cure the crystal damage and electrically activate the dopants. We define the threshold energy $E_{\mathrm{TH}}$ as the onset where the Ge surface begins to melt. It is known that changing the energy density changes the melt depth at the Ge surface. Each LTA shot covered an area of $1 \mathrm{~cm} \times 1 \mathrm{~cm}$ over 158-164 $\mathrm{ns}$ of exposure time. One P- and one As-implanted samples were subjected to RTA at $500{ }^{\circ} \mathrm{C}$ in $N_{2}$ for 3 min as a control 


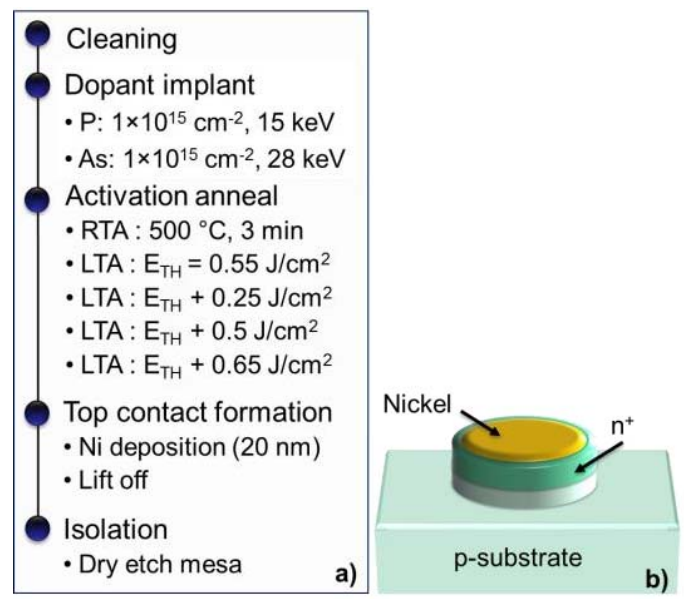

Fig. 1. (a) Summary of the experimental process flow in this paper. (b) Fabricated and characterized diode test structure.

case. Diodes were subsequently fabricated from P-implanted samples. The top contact was formed by evaporation of $20 \mathrm{~nm}$ nickel and then patterned by a liftoff process. The diodes were isolated from each other by dry etching of a mesa with 600-700 nm height [Fig. 1(b)]. No germanidation annealing was performed. Ioannou-Sougleridis et al. [15] reported enhanced leakage current caused by diffusion of Pt atoms into the depletion region during germanide formation. Fig. 1(b) shows a schematic of the fabricated and characterized circular diodes ranging from 100 to $500 \mu \mathrm{m}$ diameter.

The first stage of material characterization involved examination of the surface topography. Atomic force microscopy (AFM) was performed in noncontact tapping mode at room temperature on $5 \mu \mathrm{m} \times 5 \mu \mathrm{m}$ area for surface examination and surface roughness measurements (RMS) before and after the annealing process. Scanning electron microscopy (SEM) was performed on an FEI 650 FEG SEM to inspect the surfaces and test structures. Then, crosssectional transmission electron microscopy (TEM) was carried out to monitor the crystal integrity by using a $200 \mathrm{keV}$ JEOL 2010-HC TEM for the defect analysis, performed under weak beam dark field (WBDF) conditions, and a $200 \mathrm{keV}$ JEOL 2010F for high-resolution imaging. For carrier profiling, secondary ion mass spectrometry (SIMS) was done on a CAMECA IMS 4FE6 system, available at the UMS-CNRS Castaing caracterisation centre of Toulouse, to obtain the chemical concentration of the dopants, and electrochemical capacitance voltage (ECV) profiling was performed to determine active carrier concentration of the samples. The last stage of material characterization concerned Hall sheet carrier concentration $\left(N_{\mathrm{HS}}\right)$, mobility $(\mu \mathrm{H})$, and sheet resistance $\left(R_{S}\right)$ measurements which was done using a microHALL-M300 tool at CAPRES A/S. Several measurements were acquired on each sample for statistical average values.

\section{RESULTS AND DISCUSSION}

\section{A. Material Characterization}

The samples were initially examined with AFM to inspect the effect of LTA on the surface morphology. After implantation, the RMS was approximately $0.3 \mathrm{~nm}$.

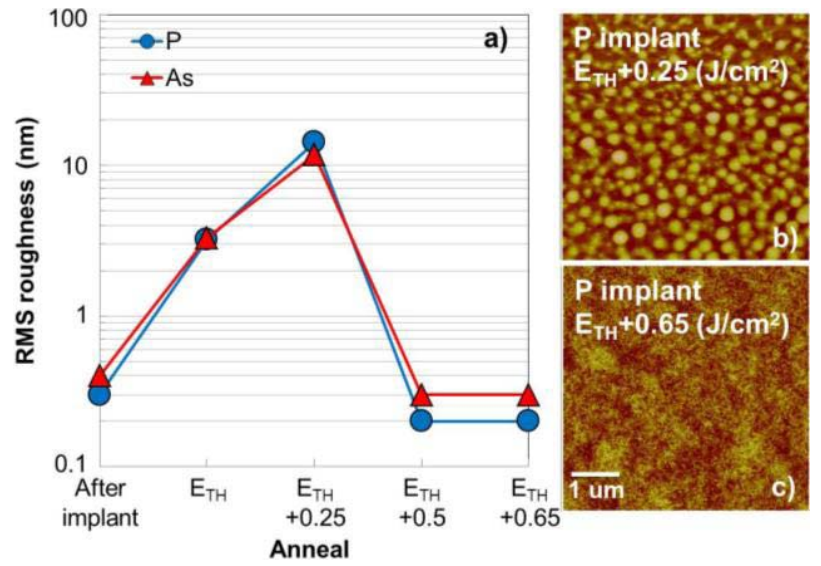

Fig. 2. (a) Surface roughness versus laser energy for $P$ and As samples. Example AFM images of P-implanted samples treated by LTA at (b) $E_{\mathrm{TH}}+0.25 \mathrm{~J} / \mathrm{cm}^{2}$ and (c) $E_{\mathrm{TH}}+0.65 \mathrm{~J} / \mathrm{cm}^{2}$.

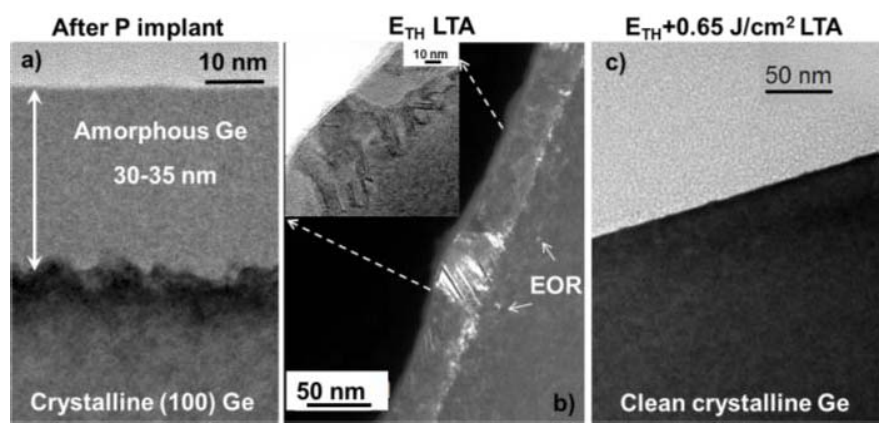

Fig. 3. Representative TEM images of (a) amorphous and crystalline interface in Ge with 30-35 nm amorphous depth after P implant, (b) defective crystalline Ge after $E_{\mathrm{TH}}$ LTA, and (c) crystalline Ge with no visible defects after $E_{\mathrm{TH}}+0.65 \mathrm{~J} / \mathrm{cm}^{2}$ LTA.

As is reflected in the extracted RMS values shown in Fig. 2(a), the application of LTA in the partial melt regime $\left(E_{\mathrm{TH}}\right.$ and $E_{\mathrm{TH}}+0.25 \mathrm{~J} / \mathrm{cm}^{2}$ ) deteriorated the roughness of the surface. It is known that these energy densities can melt in part the amorphous Ge [7]. At $E_{\mathrm{TH}}+0.5$ and $E_{\mathrm{TH}}+0.65 \mathrm{~J} / \mathrm{cm}^{2}$ the substrate was melted beyond the amorphous region which resulted in a smooth surface after the LTA process. Fig. 2(b) and (c) shows representative AFM images from P-implanted samples after $E_{\mathrm{TH}}+0.25 \mathrm{~J} / \mathrm{cm}^{2}$ and $E_{\mathrm{TH}}+$ $0.65 \mathrm{~J} / \mathrm{cm}^{2}$, respectively.

From these data, it appears that the higher LTA energy densities are more favorable to reduce the roughness of the surface.

Representative cross-sectional TEM images of the samples are shown in Fig. 3. Fig. 3(a) shows the sample after $\mathrm{P}$ implantation with the a/c interface $35 \mathrm{~nm}$ below the surface. Fig. 3(b) shows the P-implanted sample after $E_{\mathrm{TH}}$ LTA. As seen in the WBDF image, the previous amorphous layer is crystallized but stacking faults are also formed (as shown in the high-resolution image in the inset) because of poor templated recrystallization. Moreover, the presence of end-of-range (EOR) defects at $40 \mathrm{~nm}$ below the surface, behind the previous a/c interface, indicates that the melting arrived just in correspondence of the a/c interface. The 


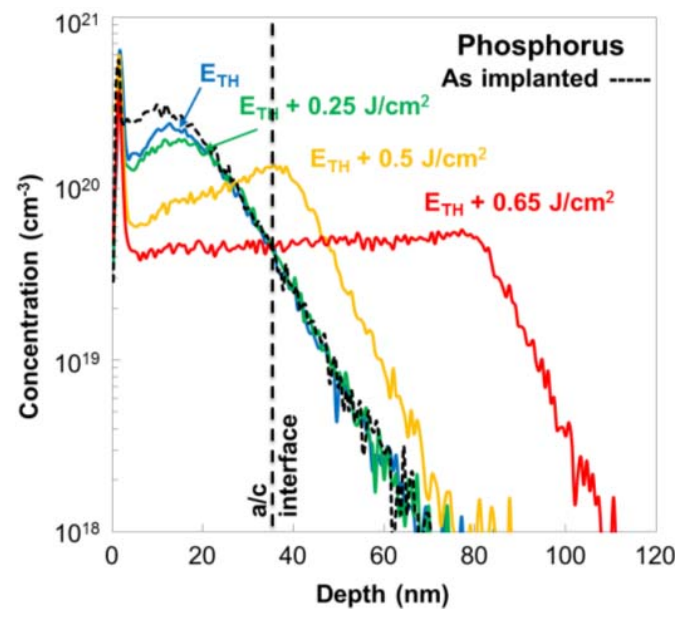

Fig. 4. SIMS profile of P-implanted samples subjected to LTA at energies ranging from $E_{\mathrm{TH}}$ to $E_{\mathrm{TH}}+0.65 \mathrm{~J} / \mathrm{cm}^{2}$.

same phenomenon was observed by Tsouroutas et al. [6]. Fig. 3(c) shows the P-implanted sample subjected to $E_{\mathrm{TH}}+$ $0.65 \mathrm{~J} / \mathrm{cm}^{2}$ LTA which resulted in apparently defect-free crystalline Ge. At this energy, Ge is melted beyond the $\mathrm{a} / \mathrm{c}$ interface.

Once again it appears that the higher thermal budget of the high energy density LTA process is desirable, as fewer crystal defects are evident in the TEM images.

Fig. 4 shows SIMS depth profiles of the P-implanted samples subjected to LTA. The a/c interface is shown by a dashed line. The peak on the surface is probably due to a SIMS artifact. From dose integration of the SIMS profiles, it was determined that $23 \%-38 \%$ of P out-diffused during the $E_{\mathrm{TH}}$ to $E_{\mathrm{TH}}+0.65 \mathrm{~J} / \mathrm{cm}^{2}$ LTA. Maximum dopant concentration of $2.4 \times 10^{20} \mathrm{~cm}^{-3}$ was obtained after LTA at $E_{\mathrm{TH}}$. No evidence of redistribution of dopants for the lowest thermal budget cases $\left(E_{\mathrm{TH}}\right.$ and $\left.E_{\mathrm{TH}}+0.25 \mathrm{~J} / \mathrm{cm}^{2}\right)$.

As expected, higher laser energies resulted in a deeper junction and more diffusion of dopants into the substrate. Enhanced redistribution of the dopants in molten Ge created a boxlike profile after $E_{\mathrm{TH}}+0.65 \mathrm{~J} / \mathrm{cm}^{2}$ LTA. In this case, the maximum dopant concentration is reduced down to $5.3 \times$ $10^{19} \mathrm{~cm}^{-3}$ owing to diffusion.

Dopant loss could be reduced significantly with a careful optimization of the implant conditions, with a much shallower amorphized depth that requires a shallower melting depth and shorter melting time, which subsequently reduces the out diffusion of dopants. Using a capping layer would also help to reduce the dopant loss, hence maximizing the active carrier concentration. An extensive study by Impellizzeri et al. [16] on the effect of LTA on activation of $B$ in Ge proved that incorporation of oxygen coming from the native oxide on the Ge surface can impede electrical activation of dopants, which could also be a concern for leakage if oxygen reaches the depletion region of the junction. Comparison of the equilibrium diffusion coefficient for oxygen in Ge [17] with that of for $\mathrm{P}$ and As extracted by Chui et al. [18] shows that $\mathrm{P}$ and As diffuse faster than oxygen in Ge during the annealing process. For the nonequilibrium

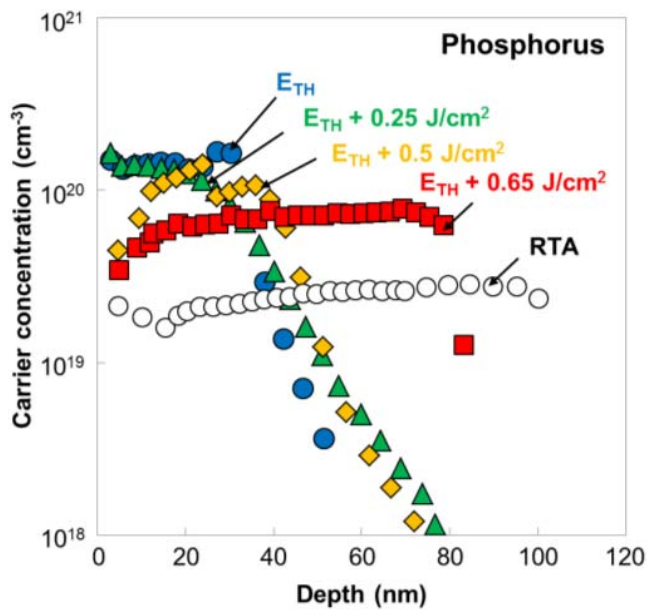

Fig. 5. Active carrier concentration from P-implanted samples obtained by ECV. LTA resulted in higher carrier concentration compared with RTA.

LTA process, we would not expect the oxygen to diffuse faster than the $\mathrm{P}$ and As, effectively catching up with the dopants and reaching the depletion region in significant concentration levels.

Fig. 5 shows active dopant concentration versus depth obtained from ECV measurements for P-implanted samples. Comparison of ECV and SIMS profiles for each of the laser energies shows a good agreement between the two techniques. Therefore it appears, within the error bars of the two characterization techniques, all the retained dose of $\mathrm{P}$ is activated, with a maximum active concentration of $1.65 \times 10^{20} \mathrm{~cm}^{-3}$, close to the result reported by Mazzocchi et al. [7] $\left(1.2 \times 10^{20} \mathrm{~cm}^{-3}\right)$ for $\mathrm{P}$ by using similar laser energy densities. Using LTA on Sb-implanted Ge, Thareja et al. [8] also achieved carrier concentrations above $10^{20} \mathrm{~cm}^{-3}$.

The observed peak at $\sim 35 \mathrm{~nm}$ in the $E_{\mathrm{TH}}$ LTA is located in the most defective zone of this sample, as found by TEM analysis [Fig. 3(b)] and indicates that the carrier concentration at this depth exceeds the dopant concentration (see SIMS profile, Fig. 4). Similar to what has already been found during $B$ activation in $\mathrm{Si}$ after excimer laser annealing [19], it is therefore suggested that the presence of this ECV peak is due to the existing defects rather than to the active dopants. Indeed, the charges measured by ECV are associated with the energy levels distributed in a wide energy range of the bandgap, including those associated with extended defects [20], [21]. Finally, from the ECV profile of the RTA control sample it can be confirmed that RTA resulted in a lower level of active concentration of $2.7 \times 10^{19} \mathrm{~cm}^{-3}$ and a deeper junction compared to the LTA samples.

Fig. 6 shows SIMS depth profiles of the As-implanted samples subjected to LTA. Here the $\mathrm{a} / \mathrm{c}$ interface lays 35-40 $\mathrm{nm}$ below the surface indicated by the dashed line. Almost no redistribution of dopants is observed at $E_{\mathrm{TH}}$ LTA. Dose integration of the SIMS profile at this energy shows no dopant loss during the LTA process. However, subsequent LTA energies resulted in 10\%-20\% dose loss. Using $E_{\mathrm{TH}}+0.65 \mathrm{~J} / \mathrm{cm}^{2}$ LTA a boxlike profile is formed with the melt depth lying about $80 \mathrm{~nm}$ below the surface. Here the 


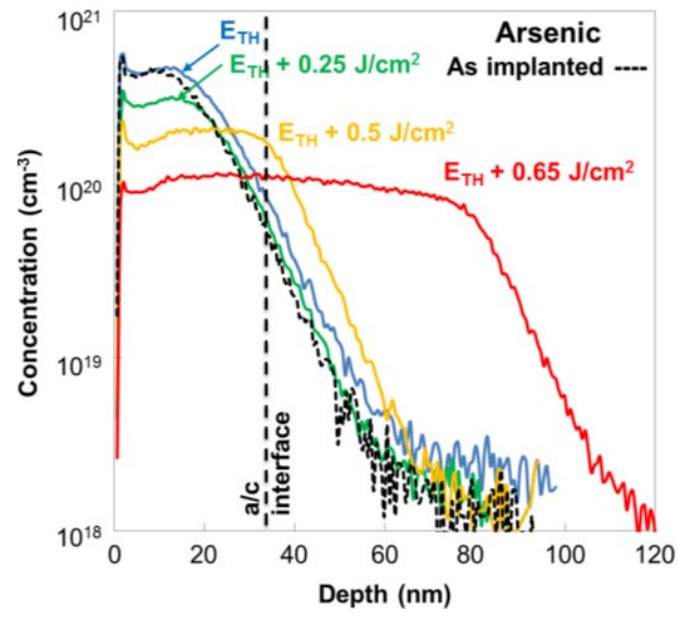

Fig. 6. SIMS profile of As-implanted samples subjected to LTA at energies ranging from $E_{\mathrm{TH}}$ to $E_{\mathrm{TH}}+0.65 \mathrm{~J} / \mathrm{cm}^{2}$.

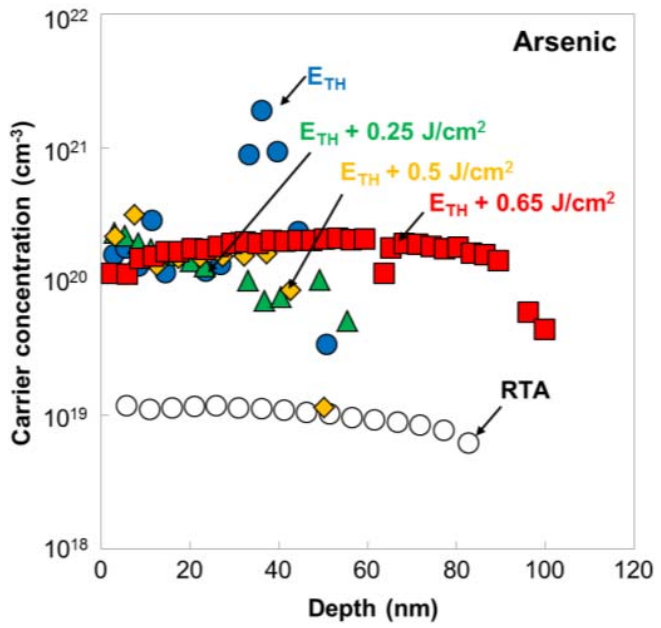

Fig. 7. Active carrier concentration from As-implanted samples obtained by ECV. LTA resulted in higher carrier concentration compared with RTA. The peak in the carrier concentration of the $E_{\mathrm{TH}}$ profile is due to the accumulation of defects in the junction not dopants alone.

plateau concentration is $>10^{20} \mathrm{~cm}^{-3}$. Similar to the $\mathrm{P}$ samples no observable defects were formed after the amorphized Ge region (including EOR defects) was fully melted and regrown.

Active carrier concentration of the As-doped samples was also measured using the ECV profiling technique as shown in Fig. 7. High active concentrations $>10^{20} \mathrm{~cm}^{-3}$ were achieved after $E_{\mathrm{TH}}$ LTA on As-implanted samples with a peak concentration of $10^{21} \mathrm{~cm}^{-3}$ at $\sim 35 \mathrm{~nm}$. Several $E_{\mathrm{TH}}$ samples were remeasured to confirm these results. Integrated dose of the $E_{\mathrm{TH}}$ profile is $>10^{15} \mathrm{~cm}^{-2}$ indicating that the charge is coming not from the dopant but from defects at the original a/c interface similar to what previously discussed for the P-implanted samples (Fig. 5).

As shown for $\mathrm{P}$ in Fig. 3, similar trends were obtained for As resulting from the LTA process. Like the $\mathrm{P}$ case, higher laser energies resulted in deeper doping profiles and lower levels of dopant concentration owing to diffusion. Again within the error bars of the ECV and SIMS characterization techniques,
TABLE I

Micro-Hall Effect Measurements

\begin{tabular}{ccccc}
\hline \hline Dopant & $\begin{array}{c}\text { Anneal } \\
\left(\mathrm{J} / \mathrm{cm}^{2}\right)\end{array}$ & $\begin{array}{c}\mu_{\mathrm{H}} \\
\left(\mathrm{cm}^{2} / \mathrm{Vs}\right)\end{array}$ & $\begin{array}{c}\mathrm{N}_{\mathrm{HS}} \\
\left(\mathrm{cm}^{-2}\right)\end{array}$ & $\begin{array}{c}\mathrm{R}_{\mathrm{s}} \\
(\Omega)\end{array}$ \\
\hline $\mathrm{P}$ & $\mathrm{E}_{\mathrm{TH}}+0.5$ & 118 & $5.78 \times 10^{14}$ & 91.8 \\
\hline $\mathrm{P}$ & $\mathrm{E}_{\mathrm{TH}}+0.65$ & 161 & $5.60 \times 10^{14}$ & 69.4 \\
\hline $\mathrm{As}$ & $\mathrm{E}_{\mathrm{TH}}+0.5$ & 94 & $9.01 \times 10^{14}$ & 73.9 \\
\hline $\mathrm{As}$ & $\mathrm{E}_{\mathrm{TH}}+0.65$ & 112 & $8.63 \times 10^{14}$ & 64.5 \\
\hline
\end{tabular}

it appears that all the retained dose of As is activated. As a comparison, Hellings et al. [3] reported 13\%-26\% activation for As-implanted Ge by using millisecond laser annealing. ECV characterization on the control RTA sample shows carrier concentration $\sim 10^{19} \mathrm{~cm}^{-3}$. Using excimer laser annealing, Milazzo et al. [5] obtained active carrier concentration of As dopant above $10^{20} \mathrm{~cm}^{-3}$.

Table I shows the mobility and micro-Hall effect measurements on P- and As-implanted samples after $E_{\mathrm{TH}}+0.5$ and $E_{\mathrm{TH}}+0.65 \mathrm{~J} / \mathrm{cm}^{2}$ LTA. Higher laser energy in both sets of samples resulted in improved carrier mobility $\left(\mu_{H}\right)$ and also lower sheet resistance $\left(R_{S}\right)$. This is consistent with the observations from material studies where the higher laser energy repaired the crystal lattice after the implantation process (Fig. 3). Extracted sheet carrier densities with Hall measurements $\left(N_{\mathrm{HS}}\right)$ is in good agreement with the active integrated concentrations obtained using ECV profiling technique. Active carrier concentration normally increases with increased thermal budget but from these measurements it remains constant. The observed discrepancy in the extracted values may be associated with the out-diffusion of the dopants during the thermal treatment as well as an almost perfect activation of dopants in both cases.

In conclusion, from the material analysis, it is apparent that the best choice of LTA condition depends on a number of factors. At low energy density, we observe a diffusionless high activation of dopants, at a cost of residual defects in the crystal. At high energy density, crystalline integrity is drastically better while maintaining very impressive levels of carrier concentration. However, care must be taken to optimize the process to avoid excessive dopant diffusion.

\section{B. Electrical Characterization}

Fig. 8 shows $I-V$ characteristics obtained from $500 \mu \mathrm{m}$ circular diodes processed by LTA and RTA. Because of the similarity in the material characterization trends for $\mathrm{P}$ and As implants, we focus on $\mathrm{P}$ in the electrical analysis in the remainder of this paper.

The $E_{\mathrm{TH}}$ LTA sample shows poor $I-V$ characteristics with very high leakage current and ideality factor $n$ larger than 2. This can be associated with the formation of a defective substrate during the recrystallization process. Annihilation of defects at higher laser energies (Fig. 3) resulted in improved $I-V$ characteristics. $I_{\mathrm{ON}} / I_{\mathrm{OFF}}$ ratio $>10^{4}$ was obtained between -1 and $1 \mathrm{~V}$ for $E_{\mathrm{TH}}+0.65 \mathrm{~J} / \mathrm{cm}^{2}$, with $n=1.4$. Note that smaller dimension diodes produced a higher $I_{\mathrm{ON}} / I_{\mathrm{OFF}}$ ratio above $10^{5}$ with $n=1.2$ (Fig. 14). 


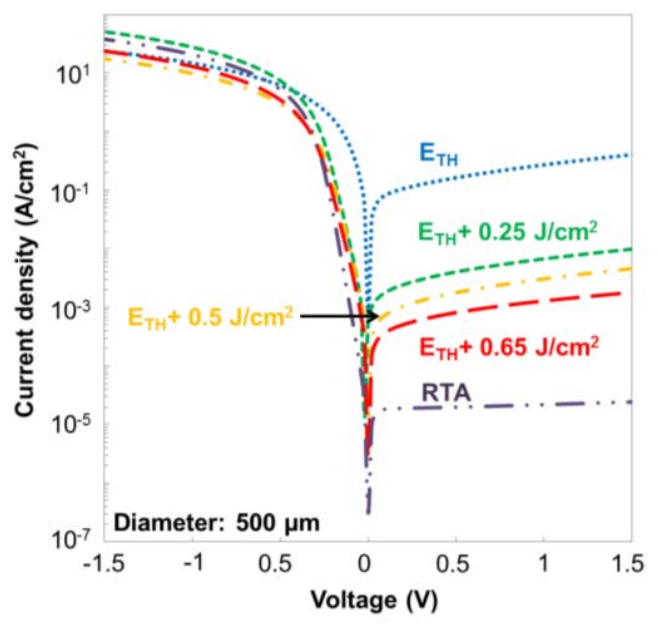

Fig. 8. Diode $I-V$ characteristics from P-implanted samples treated by RTA and LTA $\left(E_{\mathrm{TH}}-E_{\mathrm{TH}}+0.65 \mathrm{~J} / \mathrm{cm}^{2}\right)$.

Significantly, RTA diodes showed ideal behavior with $n=1.03$ and $I_{\mathrm{ON}} / I_{\mathrm{OFF}}$ ratio $>10^{6}$. From the $I-V$ characteristics, it can be concluded that there are deep level defects in the $\mathrm{n}+/ \mathrm{p}$ junctions formed by LTA which form generation centers contributing to the leakage current [22]. In the RTA case, on the contrary, long thermal budget cured the defects in the depletion layer and resulted in very low leakage current. Moreover, diffusion of dopants due to the long RTA (compared with LTA) shifted the junction depth beyond the original defective region within the $\mathrm{n}+$ part of the junction so that the remaining defects (if any) would not be located in the depletion region and have an impact on the leakage current. As stated earlier, $I-V$ measurements were carried out on a large set of diode structures from which perimeter leakage current density $\left(J_{P}\right)$ was extracted using

$$
I=P \cdot J_{P}+A \cdot J_{A}
$$

which becomes

$$
\frac{I}{A}=\frac{P}{A} \cdot J_{P}+J_{A}
$$

where $P$ and $A$ are, respectively, perimeter and area of the diode, and $J_{P}$ and $J_{A}$ are, respectively, perimeter and area leakages. To confirm perimeter leakage is not significant, Fig. 9 shows leakage current density at $1 \mathrm{~V}$ versus different perimeter/area ratios for the LTA and RTA diodes. For clarity, data are presented in semi-log scale. A straight line can be well fitted to the data in linear scale with the slope of the line corresponding to the perimeter leakage current, according to (2). It can be confirmed that both RTA and LTA diodes exhibit very low perimeter leakage which is at least two orders of magnitude smaller than the area leakage current. The graph also gives good overview of the current density for all types of samples showing improvement in leakage current from $E_{\mathrm{TH}}$ to $E_{\mathrm{TH}}+0.65 \mathrm{~J} / \mathrm{cm}^{2}$ to the RTA diode.

There are different mechanisms responsible in leakage current which are temperature and electric field dependent, and can be classified as Shockley-Read-Hall (SRH) generation/recombination, trap-assisted tunneling (TAT),

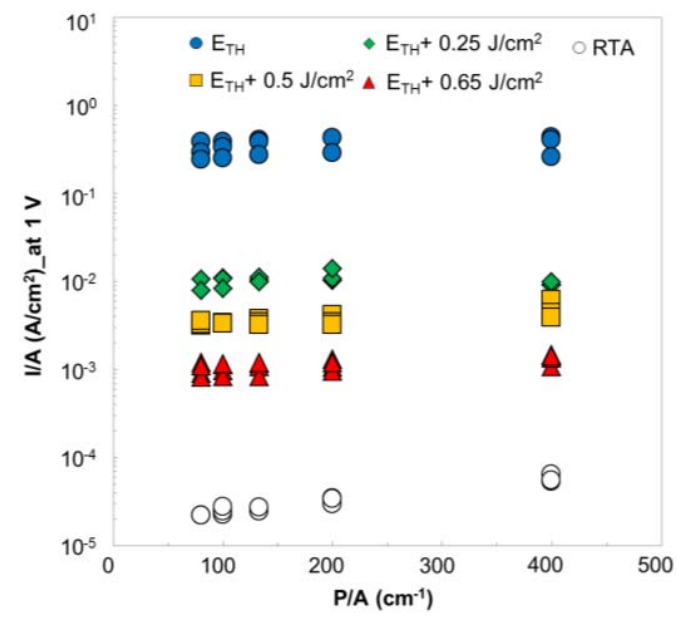

Fig. 9. Representative perimeter leakage current density at reverse voltage of $1 \mathrm{~V}$ for different perimeter area ratios for LTA and RTA diodes.

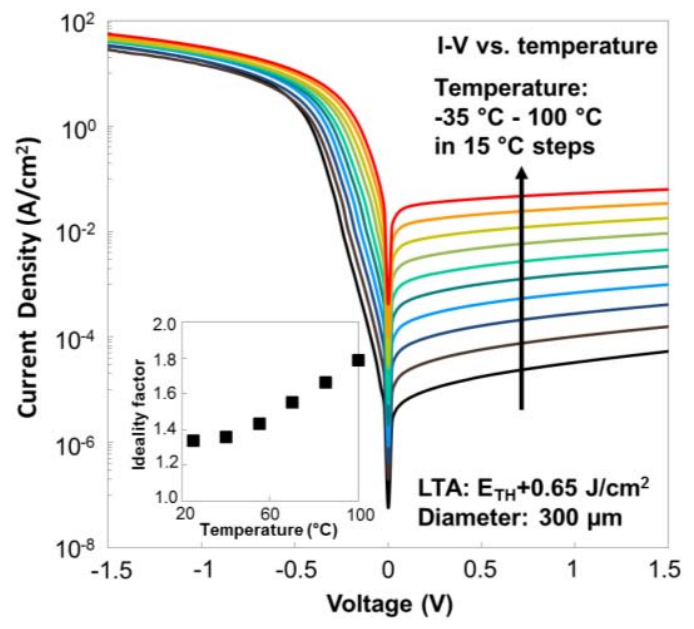

Fig. 10. $I-V$ characteristic of $E_{\mathrm{TH}}+0.65 \mathrm{~J} / \mathrm{cm}^{2}$ LTA diode for temperature increments from $-35^{\circ} \mathrm{C}$ to $100{ }^{\circ} \mathrm{C}$ in $15^{\circ} \mathrm{C}$ steps.

band-to-band tunneling (BBT) and diffusion current [22]. To determine the dominant mechanism, $I-V$ measurements were carried out at temperatures ranging from $-35{ }^{\circ} \mathrm{C}$ to $100{ }^{\circ} \mathrm{C}$ in $15^{\circ} \mathrm{C}$ steps. An activation energy $\left(E_{A}\right)$ was then derived from Arrhenius plots. Generally, SRH dominated leakage current is more pronounced in the presence of deep-level defects in the junction with $E_{A}$ approximately half of the bandgap. TAT occurs when defects operate as trap levels in the depletion region, causing electrons to be captured and tunnel due to the electric field. $E_{A}$ around half of the bandgap is also expected for TAT. If a high electric field exists in the junction, then the most probable mechanism to happen is BBT, as electrons are actuated from valence band in the p-side to the conduction band in the n-side through the depletion region. $E_{A}$ is approximately $0 \mathrm{eV}$ in this case. For diffusion current, which is proportional to the square of intrinsic carrier concentration, $\left(n_{i}^{2}\right), E_{A}$ is close to the bandgap [23].

In Fig. 10, $I-V$ characteristics from $300 \mu \mathrm{m} E_{\mathrm{TH}}+$ $0.65 \mathrm{~J} / \mathrm{cm}^{2}$ LTA diode structures at different temperatures 


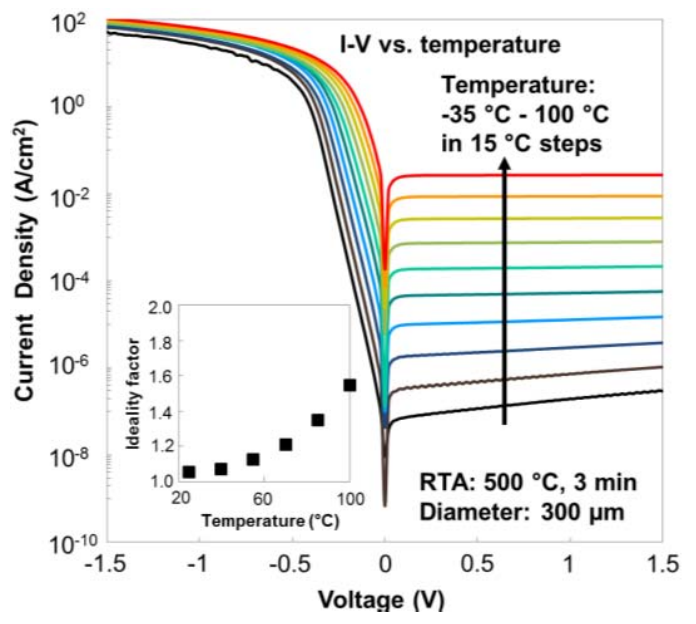

Fig. 11. $I-V$ characteristic from RTA sample for temperature increments from $-35{ }^{\circ} \mathrm{C}$ to $100{ }^{\circ} \mathrm{C}$ in $15^{\circ} \mathrm{C}$ steps.

are shown. The effect of temperature on diode behavior was reflected in the ideality factor extracted at each temperature. The inset of Fig. 10 shows variation of $n$ versus temperature with $\mathrm{n}$ increasing by temperature. In these measurements, leakage current increased about three orders of magnitude at $1 \mathrm{~V}$, suggesting that diode behavior is strongly affected by temperature variations. Meanwhile, at low temperatures [from $-35{ }^{\circ} \mathrm{C}$ to room temperature (RT)] leakage current is more dependent on the electric field.

Activation energy for this sample at $1 \mathrm{~V}$ was found to be $0.47 \mathrm{eV}$, which is bigger than half of the Ge bandgap. According to theory, SRH-dominated leakage current is proportional to depletion width which itself is proportional to $V^{0.5}$ [22]. Measurements on the LTA diode revealed that the leakage current is proportional to $V^{x}$ with $x>0.5$ for temperatures from $-35{ }^{\circ} \mathrm{C}$ to $55{ }^{\circ} \mathrm{C}$ and $x<0.5$ for temperatures from $55{ }^{\circ} \mathrm{C}$ to $100{ }^{\circ} \mathrm{C}$. These results along with extracted $E_{A}$ values, which will be shown later, point out that the leakage mechanisms are dominated by TAT and SRH, respectively.

Fig. 11 shows representative $I-V$ characteristics obtained from a $300 \mu \mathrm{m}$ RTA diode. Again, temperature measurements were carried out from $-35{ }^{\circ} \mathrm{C}$ to $100{ }^{\circ} \mathrm{C}$ with $15{ }^{\circ} \mathrm{C}$ increments. Similar to the LTA diode, ideality factor was increased by temperature as shown in the inset of Fig. 11. Leakage current was enhanced about five orders of magnitude at $1 \mathrm{~V}$ owing to the temperature change. For this particular sample, the current can be categorized in two different regimes in terms of the leakage current behavior. From $-35^{\circ} \mathrm{C}$ up to $10{ }^{\circ} \mathrm{C}$, the leakage current showed electric field dependence whereas it showed weak dependence on the electric field from $25^{\circ} \mathrm{C}$ to $100{ }^{\circ} \mathrm{C}$. This difference was also reproduced in the activation energy extraction. Note that we performed this analysis on all the diode structures and samples, and this figure shows a representative data sample set.

Fig. 12 shows reverse current density versus $1 / \mathrm{kT}$ from a $300 \mu \mathrm{m} E_{\mathrm{TH}}+0.65 \mathrm{~J} / \mathrm{cm}^{2}$ LTA diode for RT to $100{ }^{\circ} \mathrm{C}$. Extracted activation energies from the Arrhenius plots for $0.5-2 \mathrm{~V}$ are $\sim 0.5-0.4 \mathrm{eV}$, respectively. The inset shows similar graph for $1 \mathrm{~V}$ for the RTA sample where the diode behavior splits in to two regimes. Different activation

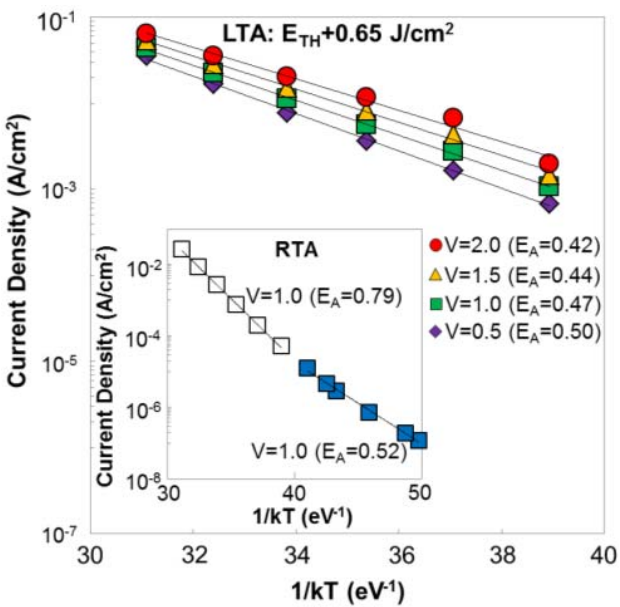

Fig. 12. Reverse current density versus $1 / \mathrm{kT}$ from $E_{\mathrm{TH}}+0.65 \mathrm{~J} / \mathrm{cm}^{2}$ LTA sample for RT and above. Activation energy is extracted from the slope of the fitted lines. Inset: current density versus 1/kT for the RTA sample for which different behavior is observed below and above RT.

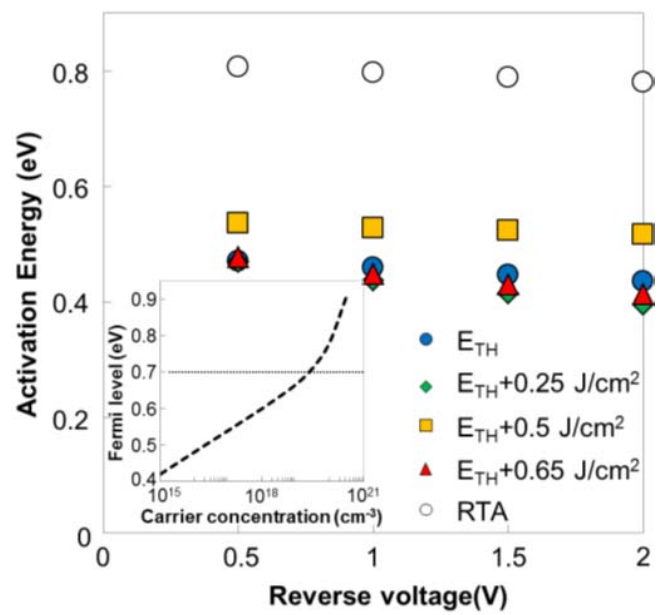

Fig. 13. Activation energy versus reverse bias extracted from RTA and LTA diodes. Inset: calculated Fermi level versus carrier concentration in the n-type region, using a 30-band k.p model.

energies at high and low temperatures were also observed by Eneman et al. [24].

Below RT, the $E_{A}$ was $0.52 \mathrm{eV}$ suggesting TAT as the main contributor to the leakage current. Interestingly, for the temperatures above RT the extracted $E_{A}$ was $0.79 \mathrm{eV}$. Considering voltage independency of the $I-V$ characteristics, it could be interpreted that diffusion is dominating the leakage mechanism in this regime with few generation centers in the depletion layer.

Activation energy versus voltage for RTA and LTA samples at RT and above is shown in Fig. 13. For all LTA samples, activation energy is between 0.4 and $0.6 \mathrm{eV}$. Note that $E_{A}=$ $0.79 \mathrm{eV}$ (RTA case) is very close to direct bandgap $(0.8 \mathrm{eV})$ of Ge. This appears to be a surprising result as the indirect bandgap of $\mathrm{Ge}$ is $0.67 \mathrm{eV}$. The reason for $E_{A}>0.67 \mathrm{eV}$ is not well understood and is currently under investigation. It should be noted that multiple samples and multiple diode structures produced this result. Furthermore, we tested our samples on two entirely different probe stations producing the same results. Moreover, we repeated the entire experiment and RTA processed diodes still produced $E_{A}>0.67 \mathrm{eV}$. 


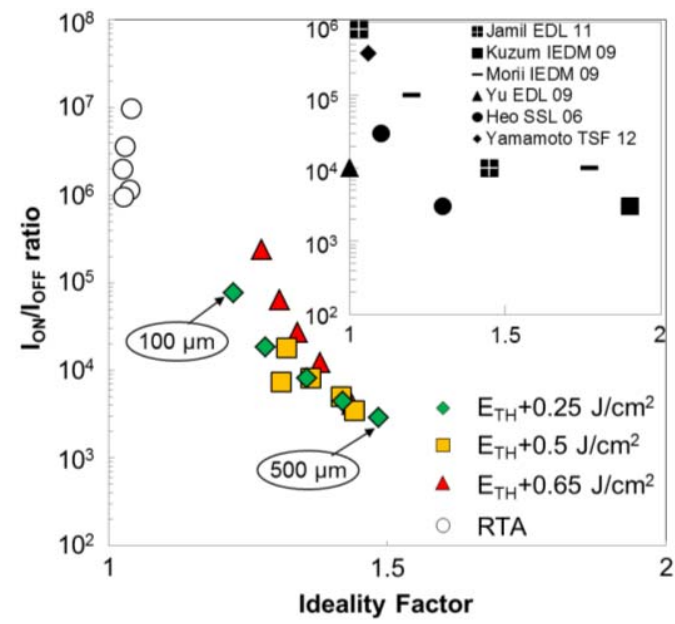

Fig. 14. $I_{\mathrm{ON}} / I_{\mathrm{OFF}}$ ratio versus ideality factor for LTA and RTA samples. Inset: $I_{\mathrm{ON}} / I_{\mathrm{OFF}}$ ratio for other works published.

The activation energies for the RTA samples are over $90 \mathrm{meV}$ in excess of what is expected for a perfectly crystalline sample doped at $2.7 \times 10^{19} \mathrm{~cm}^{-3}$. The inset in Fig. 13 shows the Fermi level versus carrier concentration for $\mathrm{Ge}$, calculated using a 30-band k.p model [25]. At the reported doping concentration, the Fermi level and hence the activation energy should lie around $0.7 \mathrm{eV}$. Possible reasons for higher activation energies are: 1) a higher carrier concentration of $\sim 10^{20} \mathrm{~cm}^{-3}$, introduced in the process of making the diodes and 2) a hybridization of the conduction band with the impurity level at high doping concentration, which may shift the bottom of the conduction band higher in energy. Both these effects would result in an $E_{A}$ strongly sensitive to doping concentration. However, we saw in our ECV data shown in Fig. 5 for RTA processed $\mathrm{P}$ implants that the active concentration was approximately $2.7 \times 10^{19} \mathrm{~cm}^{-3}$ which seems to be inconsistent with the aforementioned first explanation. We are currently investigating this trend in the experimental data further through advanced theory and modeling.

Fig. 14 shows the $I_{\mathrm{ON}} / I_{\mathrm{OFF}}$ ratio versus $n$ extracted from 100-500 $\mu \mathrm{m}$ LTA and RTA diodes. Colored symbols show the results from LTA diodes, and open symbols show the RTA case. The best $I_{\mathrm{ON}} / I_{\mathrm{OFF}}$ ratio from LTA samples was obtained from the $100 \mu \mathrm{m} E_{\mathrm{TH}}+0.65 \mathrm{~J} / \mathrm{cm}^{2}$ LTA diode with $n=1.3$ and $I_{\mathrm{ON}} / I_{\mathrm{OFF}}$ ratio $=2.4 \times 10^{5}$, which matches the best reported to date for Ge diodes with LTA [8]. RTA diodes exhibit $n$ less than 1.05 , with corresponding $I_{\mathrm{ON}} / I_{\mathrm{OFF}}$ ratio $\sim 10^{7}$, which to the best of our knowledge is the highest obtained for Ge diodes to date. $n \sim 1$ indicates that there are few defects in the junction, and the total leakage current is mainly dominated by diffusion current.

To benchmark our results, the inset shows similar works on Ge that have been reported so far [11]-[14], [26], [27]. Jamil et al. [11] used spin-on-dopant technique and reported $n \sim 1.03$ and $I_{\mathrm{ON}} / I_{\mathrm{OFF}}$ ratio $\sim 10^{5}-10^{6}$ compared with conventional ion implantation with $n=1.45$ and $I_{\mathrm{ON}} / I_{\mathrm{OFF}}$ ratio $\sim 10^{3}-10^{4}$. Kuzum et al. [12] achieved $n \sim 1.9$ and $I_{\mathrm{ON}} / I_{\mathrm{OFF}}$ ratio $\sim 10^{3}$ from nMOS devices with $\mathrm{GeO}_{x} \mathrm{~N}_{y}$ dielectrics. Morii et al. [13] reported $n \sim 1.2$ and $I_{\mathrm{ON}} / I_{\mathrm{OFF}}$ ratio $\sim 10^{5}$ from junctions created by gas phase doping and $n \sim 1.77$ and $I_{\mathrm{ON}} / I_{\mathrm{OFF}}$ ratio $\sim 10^{4}$ from P-implanted $\mathrm{n}+/ \mathrm{p}$ junctions. Yu et al. [26] compared in situ doping with ion implantation and reported $n \sim 1$ and $I_{\mathrm{ON}} / I_{\mathrm{OFF}}$ ratio $\sim 10^{3}$ for in situ doping and ion implantation, respectively. Yamamoto et al. [27] fabricated $\mathrm{n}+/ \mathrm{p}$ diodes with $n \sim 1.06$ and $I_{\mathrm{ON}} / I_{\mathrm{OFF}}$ ratio $\sim 3.7 \times 10^{5}$. Koike et al. [28] reported $I_{\mathrm{ON}} / I_{\mathrm{OFF}}$ ratio $\sim 10^{6}$ by two-step $\mathrm{P}$ implantation followed by RTA. Heo et al. [14] applied plasma doping followed by excimer laser annealing on $\mathrm{Ge}$ and obtained $n \sim 1.1-1.3$ and $I_{\mathrm{ON}} / I_{\mathrm{OFF}}$ ratio $\sim 10^{3}-10^{4}$. Their ideality factor is better than the value reported in this paper but the obtained $I_{\mathrm{ON}} / I_{\mathrm{OFF}}$ ratio seems to be lower compared with our data. In our paper, we used ion implant whereas plasma doping was used by Heo et al. [14]. This may lead to differences in damage and defect distributions close to the junction, leading to different diode performance. Plasma implant is known to reduce EOR defects and keep the implanted region very shallow $(\sim 10 \mathrm{~nm}$ in [14]). In that case, the melting time is probably shorter, thus the dopants out-diffusion is limited. In addition, high concentration of $\mathrm{P}$ on the surface could act as a capping layer limiting the oxygen incorporation from the native oxide which may result in less defects.

There is certainly a tradeoff between high levels of carrier concentration and the electrical performance of the $n+/ p$ junctions. LTA provides high carrier concentration, which is good for access resistance with tradeoff in $I_{\mathrm{ON}} / I_{\mathrm{OFF}}$. The opposite is true for RTA technique. From our data, it is evident that optimum LTA condition can be found to achieve high carrier concentration with acceptable $I_{\mathrm{ON}} / I_{\mathrm{OFF}}$ ratios.

\section{CONCLUSION}

In this paper, material and electrical characteristics of $n+/ p$ junctions in Ge formed by RTA and LTA process techniques were compared. High carrier concentration $\left(>10^{20} \mathrm{~cm}^{-3}\right)$ was accomplished using LTA for activating the dopants and also removal of the crystal damage induced by ion implantation. Retained dose drops with increasing thermal budget; however, ECV and Hall effect data indicate almost $100 \%$ activation of the dose that was retained. Furthermore, high quality $n+/ p$ junction diodes were demonstrated with $I_{\mathrm{ON}} / I_{\mathrm{OFF}}$ ratio $\sim 10^{7}$ and $I_{\mathrm{ON}} / I_{\mathrm{OFF}}$ ratio $\sim 10^{5}$ for RTA and LTA samples, respectively. To fulfill requirements for highly activated dopants and high $I_{\mathrm{ON}} / I_{\mathrm{OFF}}$ ratios, more optimization needs to be done to fine tune the LTA process. Also, more analysis is required to pinpoint the nature of the mechanism involved in extraction of activation energy greater than $0.67 \mathrm{eV}$ in RTA Ge diodes.

\section{ACKNOWLEDGMENT}

The authors would like to thank M. T. Hungria from the Centre de Microcaractérisation Raimond Castaing, Toulouse, for performing the SIMS measurements. F. Cristiano and S. Boninelli would also like to thank A. La Magna from CNR-IMM Catania for inspiring discussions.

\section{REFERENCES}

[1] K. C. Saraswat, C. O. Chui, D. Kim, T. Krishnamohan, and A. Pethe, "High mobility materials and novel device structures for high performance nanoscale MOSFETs," in Proc. Int. Electron Devices Meeting (IEDM), Dec. 2006, pp. 1-4. 
[2] J. Huang et al., "Germanium $n^{+} / p$ junction formation by laser thermal process," Appl. Phys. Lett., vol. 87, no. 17, p. 173507, 2005.

[3] G. Hellings et al., "Ultra shallow arsenic junctions in germanium formed by millisecond laser annealing," Electrochem. Solid-State Lett., vol. 14, no. 1, pp. H39-H41, 2011

[4] M. Shayesteh et al., "Atomically flat low-resistive germanide contacts formed by laser thermal anneal," IEEE Trans. Electron Devices, vol. 60, no. 7, pp. 2178-2185, Jul. 2013.

[5] R. Milazzo et al., "N-type doping of Ge by As implantation and excimer laser annealing," J. Appl. Phys., vol. 115, no. 5, p. 053501, 2014.

[6] P. Tsouroutas, D. Tsoukalas, I. Zergioti, N. Cherkashin, and A. Claverie, "Modeling and experiments on diffusion and activation of phosphorus in germanium," J. Appl. Phys., vol. 105, no. 9, p. 094910, 2009.

[7] V. Mazzocchi et al., "Boron and phosphorus dopant activation in germanium using laser annealing with and without preamorphization implant," in Proc. 17th Int. Conf. Adv. Thermal Process. Semiconductors (RTP), Sep./Oct. 2009, pp. 1-5.

[8] G. Thareja et al., "High performance germanium n-MOSFET with antimony dopant activation beyond $1 \times 1020 \mathrm{~cm}^{-3}$," in Proc. IEEE Int. Electron Devices Meeting (IEDM), Dec. 2010, pp. 10.5.1-10.5.4.

[9] K. L. Pey et al., "Formation of silicided hyper-shallow $\mathrm{p}^{+} / \mathrm{n}$-junctions by pulsed laser annealing," ECS Trans., vol. 11, no. 6, pp. 379-394, Sep. 2007.

[10] G. Eneman et al., "Impact of donor concentration, electric field, and temperature effects on the leakage current in germanium $\mathrm{p}^{+} / \mathrm{n}$ junctions," IEEE Trans. Electron Devices, vol. 55, no. 9, pp. 2287-2296, Sep. 2008

[11] M. Jamil, J. Mantey, E. U. Onyegam, G. D. Carpenter, E. Tutuc, and S. K. Banerjee, "High-performance Ge nMOSFETs with $\mathrm{n}^{+}$-p junctions formed by 'spin-on dopant'," IEEE Electron Device Lett., vol. 32, no. 9 , pp. 1203-1205, Sep. 2011.

[12] D. Kuzum, A. J. Pethe, T. Krishnamohan, and K. C. Saraswat, "Ge (100) and (111) N- and P-FETs with high mobility and low- $T$ mobility characterization," IEEE Trans. Electron Devices, vol. 56, no. 4, pp. 648-655, Apr. 2009.

[13] K. Morii, T. Iwasaki, R. Nakane, M. Takenaka, and S. Takagi, "Highperformance $\mathrm{GeO}_{2} / \mathrm{Ge}$ nMOSFETs with source/drain junctions formed by gas-phase doping," IEEE Electron Device Lett., vol. 31, no. 10, pp. 1092-1094, Oct. 2010.

[14] S. Heo et al., "Sub- $15 \mathrm{~nm} \mathrm{n}{ }^{+} / \mathrm{p}$-germanium shallow junction formed by $\mathrm{PH}_{3}$ plasma doping and excimer laser annealing," Electrochem. SolidState Lett., vol. 9, no. 4, pp. G136-G137, Apr. 2006.

[15] V. Ioannou-Sougleridis et al., "High performance $\mathrm{n}^{+} / \mathrm{p}$ and $\mathrm{p}^{+} / \mathrm{n}$ germanium diodes at low-temperature activation annealing," Microelectron. Eng., vol. 88, no. 3, pp. 254-261, 2011.

[16] G. Impellizzeri et al., "B-doping in Ge by excimer laser annealing," J. Appl. Phys., vol. 113, no. 11, p. 113505, 2013.

[17] C. Claeys and E. Simoen. (2007). Germanium-Based Technologies: From Materials to Devices. [Online]. Available: http://site.ebrary.com/ id/10172772

[18] C. O. Chui, K. Gopalakrishnan, P. B. Griffin, J. D. Plummer, and K. C. Saraswat, "Activation and diffusion studies of ion-implanted $p$ and $n$ dopants in germanium," Appl. Phys. Lett., vol. 83, no. 16, pp. 3275-3277, 2003.

[19] G. Fisicaro et al., "Kinetic Monte Carlo simulations of boron activation in implanted Si under laser thermal annealing," Appl. Phys. Exp., vol. 7 , no. 2, p. 021301, 2014.

[20] C. Nyamhere, A. Venter, F. D. Auret, S. M. M. Coelho, and D. M. Murape, "Characterization of the $\mathrm{E}(0.31)$ defect introduced in bulk n-Ge by $\mathrm{H}$ or He plasma exposure," J. Appl. Phys., vol. 111, no. 4, pp. 044511-1-044511-5, 2012.

[21] C. Nyamhere et al., "Electrical characterisation and predictive simulation of defects induced by $\mathrm{keV} \mathrm{Si}^{+}$implantation in n-type Si," J. Appl. Phys., vol. 113, no. 18, p. 184508, 2013.

[22] R. Duffy et al., "Quantitative prediction of junction leakage in bulktechnology CMOS devices," Solid-State Electron., vol. 54, no. 3, pp. 243-251, 2010

[23] R. Duffy and A. Heringa, "Characterisation of electrically active defects," Phys. Status Solidi C, vol. 11, no. 1, pp. 130-137, 2014.

[24] G. Eneman et al., "Analysis of junction leakage in advanced germanium $\mathrm{P}^{+} / \mathrm{n}$ junctions," in Proc. 37th Eur. Solid State Device Res. Conf. (ESSDERC), Sep. 2007, pp. 454-457.

[25] M. El Kurdi, G. Fishman, S. Sauvage, and P. Boucaud, "Band structure and optical gain of tensile-strained germanium based on a 30 band k.p formalism," J. Appl. Phys., vol. 107, no. 1, p. 013710, 2010.
[26] H.-Y. Yu, S.-L. Cheng, P. B. Griffin, Y. Nishi, and K. C. Saraswat, "Germanium in situ doped epitaxial growth on Si for high-performance $\mathrm{n}^{+} / \mathrm{p}$-junction diode," IEEE Electron Device Lett., vol. 30, no. 9, pp. 1002-1004, Sep. 2009.

[27] K. Yamamoto et al., "Source/drain junction fabrication for Ge metaloxide-semiconductor field-effect transistors," Thin Solid Films, vol. 520, no. 8, pp. 3382-3386, 2012.

[28] M. Koike, Y. Kamimuta, E. Kurosawa, and T. Tezuka, "NiGe/n ${ }^{+}-\mathrm{Ge}$ junctions with ultralow contact resistivity formed by two-step P-ion implantation," Appl. Phys. Exp., vol. 7, no. 5, p. 051302, 2014.

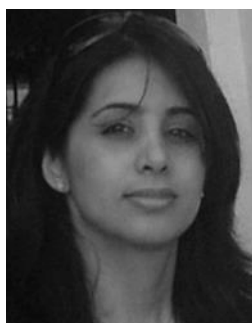

Maryam Shayesteh is currently pursuing the $\mathrm{Ph} . \mathrm{D}$. degree in novel processes, test structures, and characterization for future germanium technologies with the Tyndall National Institute, University College Cork, Cork, Ireland.

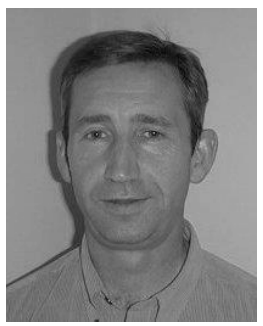

Dan O' Connell has been with the III-V Laboratory, Tyndall National Institute, University College Cork, Cork, Ireland, since 1993, where he is currently a Senior Process Engineer responsible for a wide range of thin-film deposition and patterning processes.

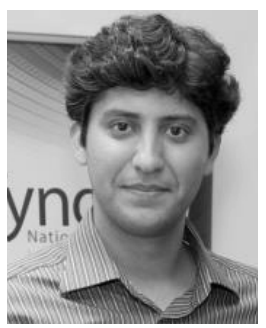

Farzan Gity (S'10) was born in London, U.K., in 1979. He received the B.S. degree from the K. N. Toosi University of Technology, Tehran, Iran, in 2003, the M.Sc. degree from Tarbiat Modares University, Tehran, in 2005, and the Ph.D. degree from the Photonics Centre, Tyndall National Institute, University College Cork, Cork, Ireland, in 2013, all in electronics engineering.

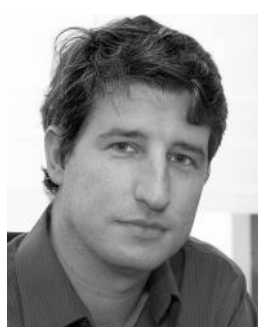

Philip Murphy-Armando is currently a Staff Researcher with the Materials Theory Group, Tyndall National Institute, University College Cork, Cork, Ireland. His current research interests include the calculation of resistivity, optical, and energy relaxation processes in materials using first principles electronic-structure theory methods.

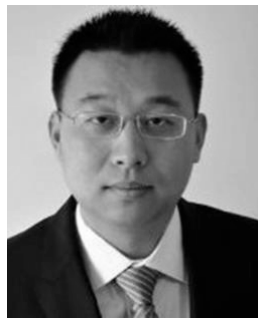

Ran Yu received the Ph.D. degree from the Tyndall National Institute, University College Cork, Cork, Ireland.

Mr. Yu is currently with Synopsys, Singapore. His current research interests include simulation, fabrication, and characterization of nanoscale transistors. 


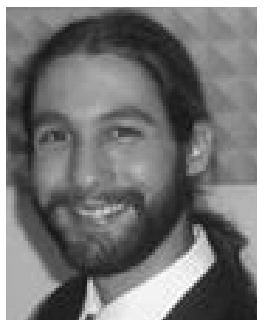

Karim Huet received the Ph.D. degree in physics from the University of Paris-Sud, Orsay, France, in 2008 , with a focus on modeling of charge transport in strained CMOS devices.

$\mathrm{He}$ is currently responsible for process and applications development involving experimental and simulation activities on laser thermal annealing with Laser Systems and Solutions of Europe, Dainippon Screen Group, Gennevilliers, France, and SCREEN Semiconductor Solutions Company, Ltd., Paris.

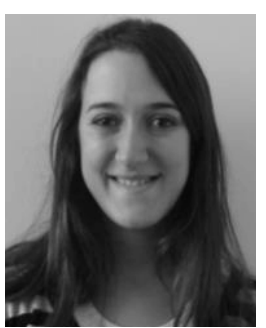

Ines Toqué-Tresonne received the Dipl.-Ing. degree in optoelectronics from the School of Engineering, Polytech Paris-Sud, Orsay, France, in 2011.

She is currently an Application Engineer, managing the Application Laboratory for Laser Thermal Annealing projects at Laser Systems and Solutions of Europe, Dainippon Screen Group, Gennevilliers, France, and SCREEN Semiconductor Solutions Company, Ltd., Paris.

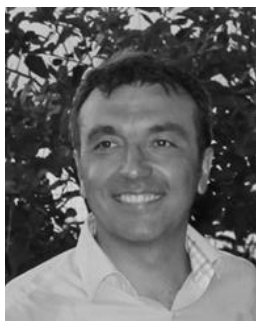

Fuccio Cristiano received the B.Sc. degree in physics from the University of Catania, Catania, Italy, in 1991, and the Ph.D. degree from the University of Surrey, Surrey, U.K., in 1998, with a focus on the synthesis and characterization of SiGe alloy layers for heterojunction bipolar transistors.

He joined the Laboratory for Analysis and Architecture of Systems, Toulouse, France, in 2000, where he is currently a Staff Scientist.

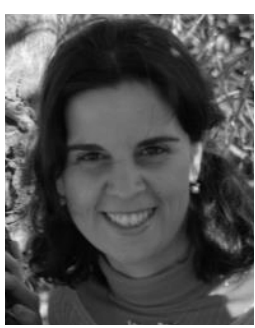

Simona Boninelli received the Ph.D. (cum laude) degree in physics from the University of Catania, Catania, Italy, in 2005, with a focus on structural characterization of silicon nanostructures for the realization of light-emitting devices.

She is currently a Permanent Researcher with the Institute of Microelectronics and Microsystems, National Research Council, University of Catania.

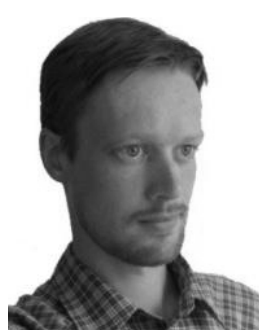

Henrik Hartmann Henrichsen received the M.Sc. degree in engineering, physics, and technology from the University of Southern Denmark, Odense, Denmark, in 2005. He is currently pursuing the Ph.D. degree with the Technical University of Denmark, Kongens Lyngby, Denmark, in 2007, with a focus on investigating electrical interfaces for organic nanodevices.

$\mathrm{He}$ is currently with CAPRES A/S, Kongens Lyngby.

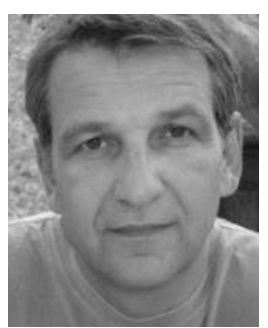

Peter Folmer Nielsen was born in Denmark in 1958.

He founded CAPRES A/S, Kongens Lyngby, Denmark, in 1999. He is currently a Chief Technology Officer and in charge of Research and Development. His current research interests include extending the applications for the unique Capres microprobe and probing tools technology.

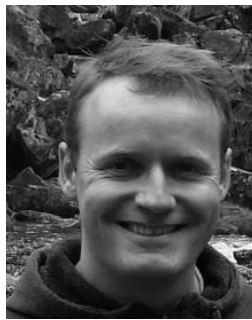

Dirch Hjorth Petersen was born in Denmark in 1979. He received the M.Sc. and Ph.D. degrees in applied physics from the Technical University of Denmark (DTU), Kongens Lyngby, Denmark, in 2006 and 2010, respectively.

$\mathrm{He}$ is currently a Senior Researcher with the Nanocarbon Group, DTU Nanotech, Kongens Lyngby. His current research interests include methods for electrical characterization of ultrathin materials.

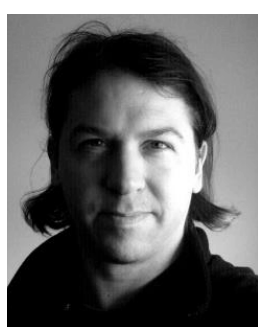

Ray Duffy is currently with the Tyndall National Institute, University College Cork, Cork, Ireland, where he is involved in material science, process optimization, modeling, and device studies in emerging devices and materials, for advanced semiconductor applications. 